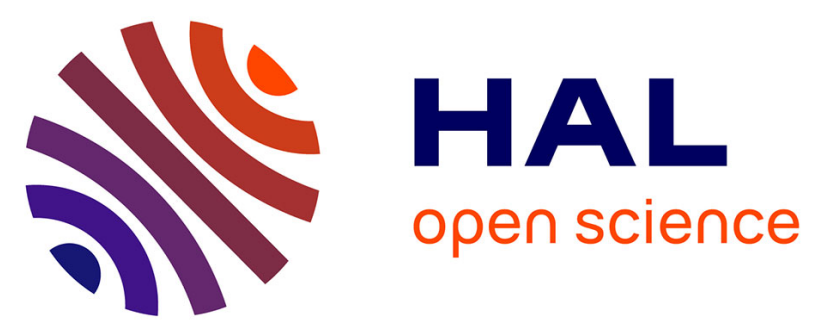

\title{
Terahertz radiation for tomographic inspection
}

Benoît Recur, Jean Paul Guillet, Léna Bassel, Carole Fragnol, Inka

Manek-Hönninger, Jean-Christophe Delagnes, William Benharbone, Pascal

Desbarats, Jean-Philippe Domenger, Patrick Mounaix

\section{- To cite this version:}

Benoît Recur, Jean Paul Guillet, Léna Bassel, Carole Fragnol, Inka Manek-Hönninger, et al.. Terahertz radiation for tomographic inspection. Optical Engineering, 2012, 51 (9), pp.091609-1 - 091609-7. 10.1117/1.OE.51.9.091609 . hal-00699947

\section{HAL Id: hal-00699947 https://hal.science/hal-00699947}

Submitted on 22 May 2012

HAL is a multi-disciplinary open access archive for the deposit and dissemination of scientific research documents, whether they are published or not. The documents may come from teaching and research institutions in France or abroad, or from public or private research centers.
L'archive ouverte pluridisciplinaire HAL, est destinée au dépôt et à la diffusion de documents scientifiques de niveau recherche, publiés ou non, émanant des établissements d'enseignement et de recherche français ou étrangers, des laboratoires publics ou privés. 


\section{Optical Engineering}

\section{Terahertz radiation for tomographic inspection}

Benoit Recur

Jean-Paul Guillet

Lena Bassel

Carole Fragnol

Inka Manek-Hönninger

Jean Christophe Delagnes

William Benharbone

Pascal Desbarats

Jean-Philippe Domenger

Patrick Mounaix 


\section{Terahertz radiation for tomographic inspection}

\section{Benoit Recur}

Université de Bordeaux

LaBRI, UMR5800 CNRS

F-33405 Talence, France

E-mail: brecur@labri.fr

\author{
Jean-Paul Guillet \\ Lena Bassel \\ Carole Fragnol \\ Inka Manek-Hönninger \\ Jean Christophe Delagnes \\ William Benharbone \\ Université de Bordeaux \\ LOMA, UMR5798 CNRS \\ F-33405 Talence, France
}

\section{Pascal Desbarats}

Jean-Philippe Domenger

Université de Bordeaux

LaBRI, UMR5800 CNRS

F-33405 Talence, France

\section{Patrick Mounaix}

Université de Bordeaux

LOMA, UMR5798 CNRS

F-33405 Talence, France

E-mail: p.mounaix@loma.u-bordeaux1.fr

\begin{abstract}
Three-dimensional (3-D) terahertz computed tomography has already been performed with three different reconstruction methods (standard back-projection algorithm and two iterative analyses) to reconstruct $3-D$ objects. A Gaussian beam model is developed according to the physical properties of terahertz waves such as the energy distribution within the propagation path. This model is included as a new convolution filter into the tomographic reconstruction methods in order to analyze the impact of a such effect and then to enhance quality and accuracy of the resulting images. We demonstrate the improvements of the optimized reconstructions for applied 3-D terahertz tomography. @ 2012 Society of PhotoOptical Instrumentation Engineers (SPIE). [DOI: 10.1117/1.OE.51.9.091609]
\end{abstract}

Subject terms: terahertz imaging; tomographic imaging; three-dimensional image processing; image reconstruction techniques; scanners.

Paper 111577SS received Dec. 15, 2011; revised manuscript received Feb. 23, 2012; accepted for publication Mar. 19, 2012; published online May 15, 2012.

\section{Introduction}

Due to their non-ionizing properties and relative transparency in most materials, terahertz radiations or T-rays are appealing for several imaging and inspection applications. ${ }^{1-3}$ However, the currently available emitter/detector sets limit for the range of applications because their limited tunability and low sensitivity lead to a prohibitive acquisition time. In the three-dimensional (3-D) imaging application field, X-ray computed tomography (CT) is an omnipresent technique that provides cross-sectional images of an object by analyzing the radiation transmitted through the sample at different incidence angles. Nevertheless, this technique cannot be easily applied to soft materials such as plastics, papers, or paintings owing to the low absorption of the $\mathrm{X}$-ray radiation.

In 2002, THz CT was proposed for 3-D imaging of opaque materials. ${ }^{4}$ Ferguson et al. demonstrated that cross-sectional images can be obtained by measuring the transmitted amplitude and phase of broadband $\mathrm{THz}$ pulses at multiple projection angles. These $\mathrm{THz}$ radiations propose attractive features such as non-invasive and non-destructive analysis, transparency, and good penetration depth through various materials, especially below $1 \mathrm{THz}$. All these remarkable properties make $\mathrm{THz}$ radiation very efficient for direct applications in non-destructive inspection and in the security field. Most of the experimental demonstrations were

0091-3286/2012/\$25.00 @ 2012 SPIE performed in two dimensions whereas for $\mathrm{THz} \mathrm{CT}$, which is a powerful technique, the literature remains scattered. ${ }^{4-9}$

Three restrictions slow down the progress of this application. First, the Fresnel diffraction effects and the multiple refraction and reflection losses experienced by the $\mathrm{THz}$ wave through the sample ${ }^{10}$ make it difficult to decide unambiguously whether the imaging contrast arises from the sample absorption caused by the wave attenuation only, the beam deviation or both. The second limitation is the long acquisition time required by pulsed $\mathrm{THz}$ systems, based on a time-domain spectrometer, since it uses a point-to-point measurement associated with temporal sampling. This limitation can be reduced by using a continuous wave $\mathrm{THz}$ source even if, in this case, the phase information of the object cannot be extracted. The last limitation is directly connected to the minimal and necessary number of acquired projections to perform a CT reconstruction without deformation.

Finally, an important consideration in CT concerns the choice of the reconstruction method to visualize the different cross-sectional images and the final 3-D volume of the sample. Usually, the back-projection of filtered projections (BFP) is employed as the standard reconstruction method. ${ }^{11}$ The analysis is based on the inverse Radon transform from the projection dataset. ${ }^{12}$ In X-ray CT, it is well known that the BFP suffers from several drawbacks such as beam hardening, noise sensitivity, and geometric degradation in case of an insufficient number of projections. Consequently, alternative iterative reconstruction methods such as the simultaneous algebraic reconstruction technique (SART) $)^{13,14}$ and the 
ordered dubsets expectation maximization (OSEM) $)^{15,16}$ have been proposed for X-ray CT.

In this paper, these three reconstruction methods (BFP, SART, OSEM) are applied to THz CT. Of particular interest is the optimization of the number of projections associated with the preservation of the image quality. At minimum, we propose to improve the physical modeling by adding an heuristic propagation of a Gaussian beam. We aim to take into account a more realistic physical behavior of the electromagnetic waves used in THz CT. This approach is justified by the fact that the reconstruction methods assume a ray tracing approach, while the $\mathrm{THz}$ beam profile is very far from this basic hypothesis. There is indeed a trade-off range in the focusing condition of the diffracting $\mathrm{THz}$ wave. While $2 \lambda$-waist beams can be qualitatively treated as a ray of light, tightly focused ones (down to the ultimate resolution of $\lambda / 2$ ) can hardly accommodate with the approach of all methods used in X-ray because the beam energy propagation is not uniform along a line anymore. Finally, the Gaussian model developed in this paper is included in the reconstruction techniques to enhance the overall quality and accuracy of resulting images.

\section{Experimental Setup Properties}

The experimental setup of the 3-D millimeter wave tomographic scanner is shown in Fig. 1(a). The output beam of a compact Gunn diode coupled with a horn antenna is collimated using an off-axis parabolic mirror. The Gunn diode used is an $84-\mathrm{GHz}$ diode coupled to a frequency tripler delivering $0.3 \mathrm{~mW}$ at $240 \mathrm{GHz}$ (wavelength $1.25 \mathrm{~mm}$ ). The $\mathrm{THz}$ beam is then focused with a PTFE lens $(f=75 \mathrm{~mm}$ focal length and $D=50.8 \mathrm{~mm}$ diameter) on the sample, which is positioned on a three-axes motorized stage comprising the $X, Y$, and $\theta$ movements, respectively. The detection is performed with a pyroelectric sensor and the beam is modulated at $20 \mathrm{~Hz}$ by an optical chopper. The amplitude of the transmitted $\mathrm{THz}$ is acquired with a lock-in amplifier. Then we can map the two-dimensional (2-D) transversal profile of the $\mathrm{THz}$ beam at the beam waist and outside the Rayleigh range. At the central sample position, we check if the beam profile is homogeneous with a Gaussian circular shape [2 $\mathrm{mm}$ beam diameter, measured at full width at half maximum (FWHM)] in agreement with the theoretical values obtained from the propagation of Gaussian beam models. This result indicates that the spatial resolution of the 3-D millimeter wave tomographic scanner is limited to a few millimeters owing to the long wavelength of the emitting sources.

The sample we analyze [Fig. 1(b)] is a polymer made of Rohacell, which is quite transparent in the lower part of the terahertz bandwidth. Its dimensions are $30 \times 34 \times 37 \mathrm{~mm}^{3}$. We inserted two metallic balls as eyes and a screw in an oblique direction inside the polymer skull. In order to perform a 3-D reconstruction of Terakenstein, we first record a 2-D transmission image of the sample by moving the object in the $X$ and $Y$ directions with a scan step of $1 \mathrm{~mm}$ in both directions. The acquisition time for a $50 \times 50$ pixel image is about $3 \mathrm{~min}$. Next, the sample is rotated by a rotation step $d \theta$ in order to provide different visualization angles of the object. The operation is repeated $N_{\theta}$ times from $\theta=0$ to $180 \mathrm{deg}$ and we finally get a set of $N_{\theta}$ projections. For instance, if $N_{\theta}=36, d \theta=5 \mathrm{deg}$, the total acquisition time is about $2 \mathrm{~h}$ for the complete 3-D visualization of the sample. From the overall projection images [Fig. 2(a)], we can extract

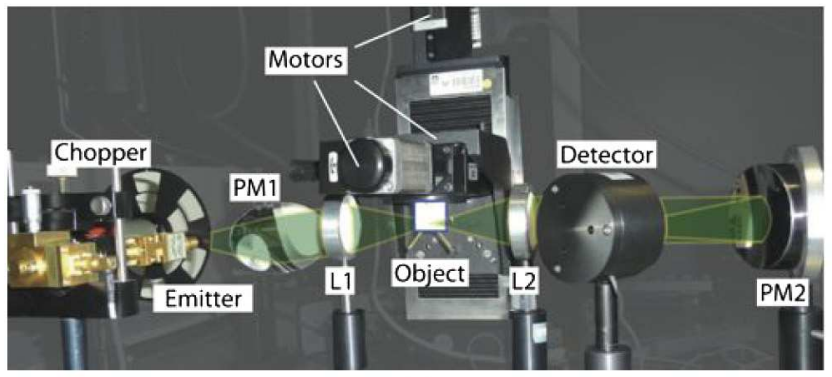

(a)

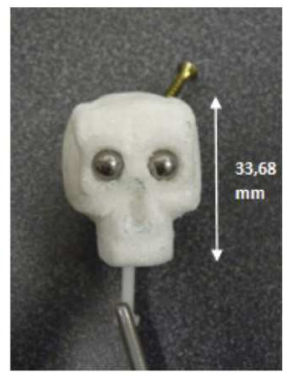

(b)

Fig. 1 (a) Experimental setup. L1-L2: PTFE lenses; PM1-PM2: parabolic mirrors. (b) The sample under investigation: Terakenstein.

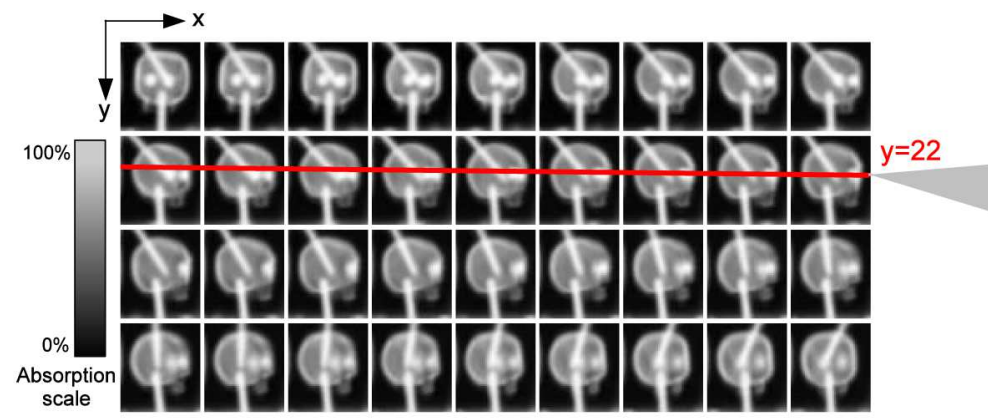

(a)

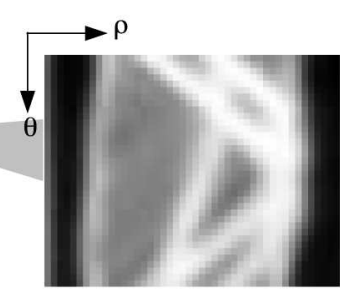

(b)

Fig. 2 (a) 2-D projections acquired between 0 and 180 with an angle step $d \theta=5$ deg. (b) Sinogram of the cross-section at position $Y=22$. 


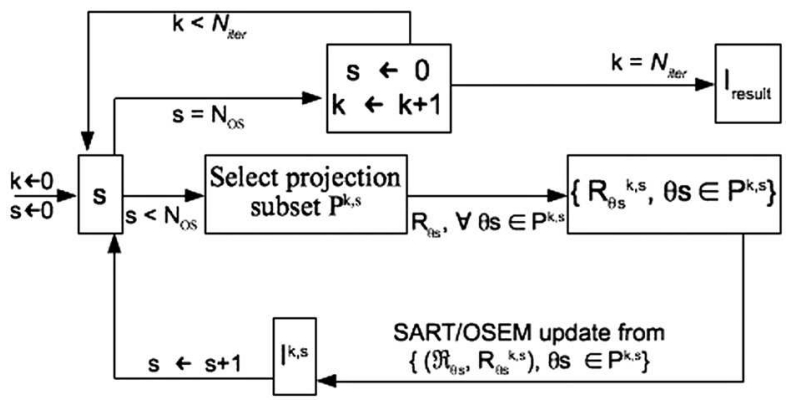

Fig. 3 Iterative reconstruction process.

a sinogram which represents, for a given horizontal slice, the evolution of the transmitted $\mathrm{THz}$ amplitude as a function of the rotation angle. For example, the sinogram in Fig. 2(b), extracted from projection images at position $Y=22$, contains $N_{\theta}=36$ projections (in rows) of $N_{\rho}=50$ samples per projection (in columns).

\section{Tomographic Reconstructions}

Mathematically, the absorption sum undergone by the ray along the line $(\theta, \rho)$ [positioned at similar coordinates in the sinogram Fig. 2(b)] is modeled by the Radon transform ${ }^{12}$ as follows:

$\mathcal{R}_{\theta}(\rho)=\int_{-\infty}^{\infty} \int_{-\infty}^{\infty} f(x, y) \delta(\rho-x \cos \theta-y \sin \theta) \mathrm{d} x \mathrm{~d} y$,

where $\theta$ and $\rho$ are respectively the angular and radial coordinates of the projection line $(\theta, \rho), \delta$ is the Dirac impulse, and $f(x, y)$ is the absorption function.

The inverse Radon transform, denoted $\mathrm{BFP},{ }^{17,18}$ is usually performed to recover the original domain from the projections. First, this inversion filters each projection in the Fourier domain to increase geometric details. Second, it computes each $f(x, y)$ value as a sum of filtered projections crossing $(x, y)$.

Apart from this direct method, iterative techniques originally developed for X-ray scan imaging, have been introduced for $\mathrm{THz} \mathrm{CT}$ imaging by Recur et al. ${ }^{18}$ For instance, SART $^{13,14}$ approaches the solution of the linear equation system $I=A^{T} R$, where $I$ is the image, $R$ is the sinogram, and $A$ is the weight-matrix using an iterative process following $k \in\left[0 \ldots N_{\text {iter }}\right]$. Each sub-iteration $s, 0 \leq s<N_{\theta}$, updates each pixel of the image $I^{k, s}$ by comparing the original projection $\mathcal{R}_{\theta_{s}}$ with $R_{\theta_{s}}^{k}$ (computed from $I^{k, s-1}$ ) as follows:

$$
I^{k, s}(i, j)=I^{k, s-1}(i, j)+\lambda \frac{\sum_{i_{\rho}=0}^{N_{\rho}-1} A_{(\theta, \rho),(i, j)}\left[\frac{\mathcal{R}_{\theta_{s}}(\rho)-R_{\theta_{s}}^{k}(\rho)}{D_{\theta_{s}}(\rho)}\right]}{\sum_{i_{\rho}=0}^{N_{\rho}-1} A_{(\theta, \rho),(i, j)}},
$$

where:

- $D_{\theta}(\rho)=\sum_{i=0}^{W-1} \sum_{j=0}^{H-1} A_{(\theta, \rho),(i, j)}$ is the norm of the segment $(\theta, \rho)$ crossing the image,

- $R_{\theta_{s}}^{k}(\rho)=\sum_{i=0}^{W-1} \sum_{j=0}^{H-1} A_{\left(\theta_{s}, \rho\right),(i, j)} I^{k, s-1}(i, j)$,

- $(W \times H)$ is the image size.

A super-iteration $k$ is completed when all the projections have been used. Iterations in $k$ are performed until the convergence of the solution is achieved. The initial $I^{0,0}$ image is usually an uniform image. The scheme in Fig. 3 summarizes the iterative method process. The update step of the algorithm depends on the iterative technique used. In the following, we consider the SART and the OSEM techniques, which only differ by the number of projection subsets and the error update estimation. The images in Fig. 4 represent the reconstructions from the projection dataset shown in Fig. 2 using the three methods. The different slices correspond to the cross-sections at 5-mm steps along the $Y$-axis. All three methods are able to resolve more or less the contours with a clearly visible contrast and reveal the inner structure of the sample.

\section{Gaussian Beam Model and Method Optimizations}

In X-ray CT transmission processes, the beam shape can be considered constant because of the smallness of the wavelength with respect to the object size so that there is no diffraction. For the same reason, the intensity distribution is uniform over the beam cross-section. This property allows the Radon model to be used directly in all reconstruction methods.

In $\mathrm{THz}$ CT imaging, the propagation beam is close to a Gaussian distribution, which is both determined by the $\mathrm{THz}$ wave properties and the lens used to enforce the beam focus. The radius of the beam (from the beam axis) has its minimum value $w_{0}$ at the beam waist. According to the wavelength $\lambda$, the radius at the position $z$ from the beam waist is:

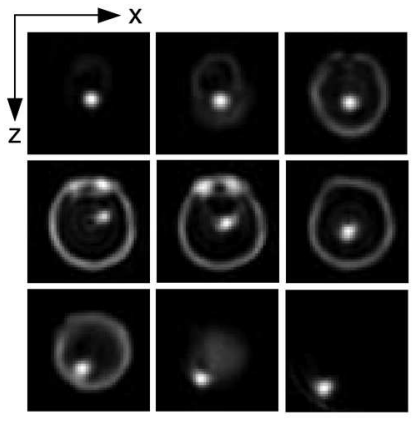

(a)

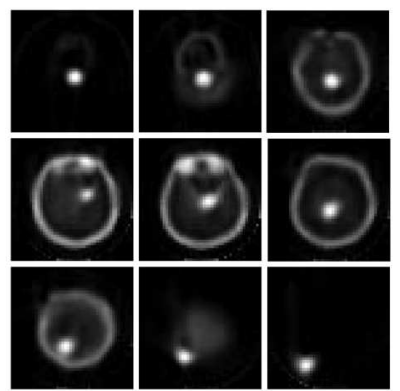

(b)

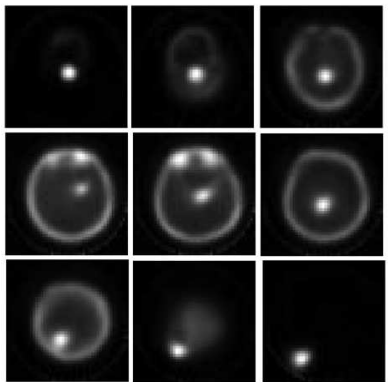

(c)

Fig. 4 Reconstructed cross-sectional images with BFP (a), SART (b), and OSEM (c) techniques, respectively. 


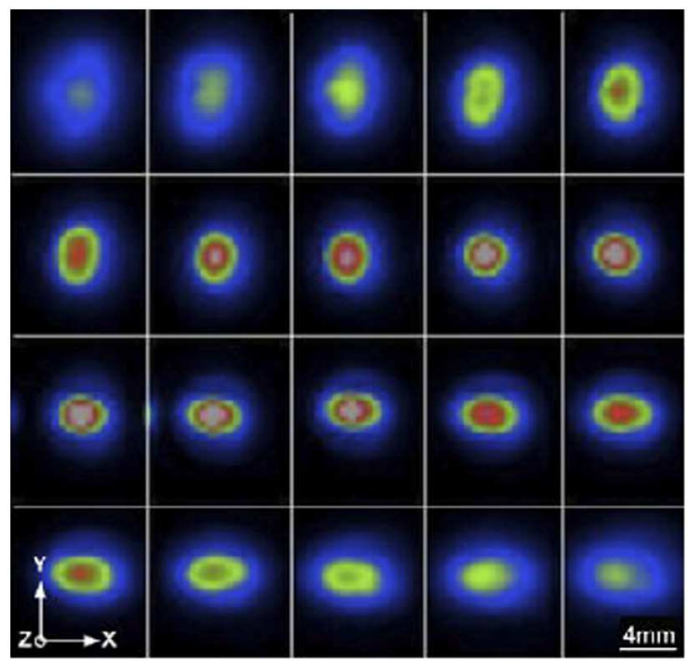

(a)

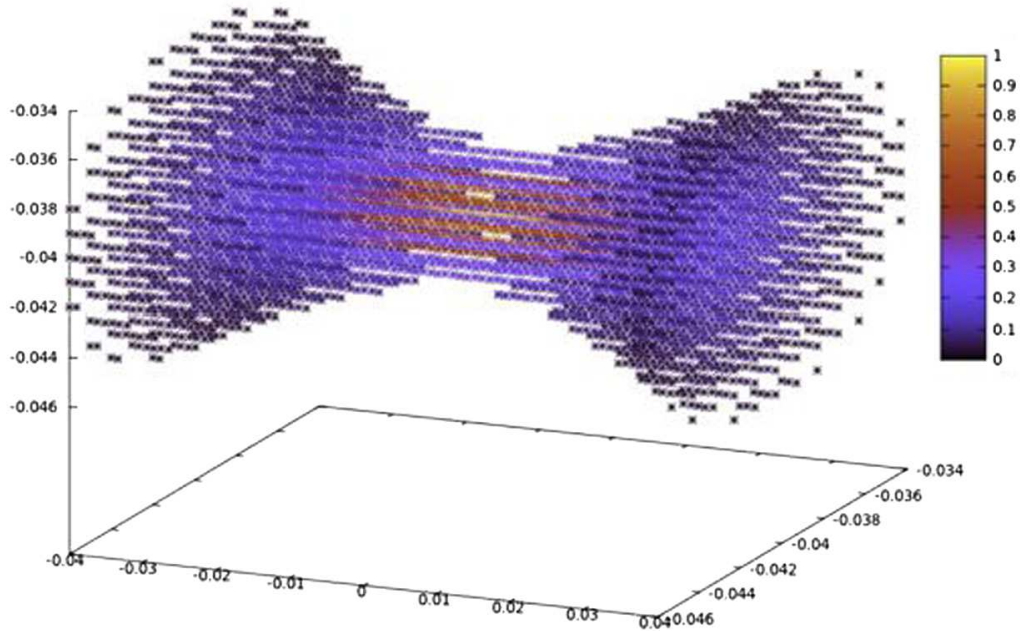

(b)

Fig. 5 (a) Propagation beam acquired along the Z-axis with a $5 \mathrm{~mm}$ step. (b) Simulated propagation of the beam for a 240-GHz source [Intensity (a.u), position in meters], FWHM $=2 \mathrm{~mm}$ at the beam waist according to the previously measured beam.

where $z_{R}=\pi w_{0}^{2} / \lambda$ is the Rayleigh range. Moreover, the intensity distribution over the cross-section is given by:

$I(r, z)=I_{0}\left[\frac{w_{0}}{w(z)}\right]^{2} \exp \left[\frac{-2 r^{2}}{w^{2}(z)}\right]$,

where:

- $\mathrm{r}$ is the orthogonal distance from the beam $Z$-axis,

- $I_{0}$ is the intensity at the center of the beam waist.

Figure 5(a) shows beam cross-sections obtained with a 240-GHz source. These 2-D images are obtained using a photothermal $\mathrm{THz}$ sensor positioned along the $Z$-axis with a displacement of $5 \mathrm{~mm}$ between each orthogonal image profile. Note that the energy decreases from the center to the edge of the beam following a Gaussian distribution. Using Eqs. (3) and (4), the observed Gaussian beam can be computed to simulate a given source. For instance, Fig. 5(b) shows the $3-\mathrm{D}$ profile and the energy distribution of a beam simulating the $240-\mathrm{GHz}$ propagation focalized FWHM $=2 \mathrm{~mm}$ (at beam waist) corresponding to the observed propagation [Fig. 5(a)].

The acquisition process through such a beam is equivalent to an acquisition of the original object convoluted by the Gaussian beam. ${ }^{19}$ Mathematically, this process is given in two dimensions by the following optimization of the Radon transform:

$$
\begin{aligned}
\mathcal{R}_{\phi=0}(\rho)= & \int_{-\infty}^{\infty} f_{\theta}(x-\rho, y) \\
& * I\left(\rho-x_{0}, y-y_{0}\right) \delta(\rho-y \cos \phi) d x,
\end{aligned}
$$

where $I(x, y)$ is the Gaussian propagation model Eq. (4), $*$ is the convolution operator, and $\left(x_{0}, y_{0}\right)$ is the center of the beam waist. The $f(x, y)$ domain is rotated by $\theta$ to assume $\phi=0$.

It leads to a horizontal acquisition allowing an easier computation of the column-by-column convolutions (in two dimensions) and $X Y$ plane-by-plane convolutions (in three dimensions).
The direct reconstruction process consists in inverting the convoluted acquisition. The projection acquired through a Gaussian beam is retroprojected into a temporary image. This image is deconvoluted column-by-column with the beam model, then rotated by the angle $-\theta$ and added to the result image.

Using iterative methods, the projection calculated through a simulated Gaussian beam at an iteration $k$ is given by the discrete version of Eq. (5). Then, the update step comparison between computed and measured acquisitions becomes consistent because the projections are both obtained through a simulated/real Gaussian beam. Moreover, similarly to BFP, the obtained error projection is deconvoluted before it is added in the $I^{k+1}$ image. Everything else in the algorithm in Fig. 3 remains the same.

\section{Results and Discussion}

The images in Fig. 6 represent the results obtained with BFP, SART, and OSEM using the Gaussian beam model in the reconstruction methods. To show the new method efficiency, quality of the results are now compared to each other using image-based comparison metrics. The quality preservation is estimated using the equivalence rate between two images. The image properties are compared to each other using the Structural SIMilarity (SSIM) criterion: ${ }^{20}$

$\operatorname{SSIM}(I, J)=l(I, J) \cdot c(I, J) \cdot r(I, J)$,

where $0 \leq l(I, J) \leq 1[\operatorname{resp} .0 \leq c(I, J) \leq 1]$ is the global intensity (resp. contrast) comparison between two images $I$ and $J$, and $0 \leq r(I, J) \leq 1$ is the correlation coefficient. $0 \leq$ $\operatorname{SSIM}(I, J) \leq 1$ gives the quality rate of $J$ compared to the reference $I$. The closer the SSIM value is to 1 , the better the reconstruction quality is.

We compare the image quality according to the number of projections. For each reconstruction methods, we choose as reference image the volume obtained from $N_{\theta}=36$ projections. Reconstructions using a smaller projection number $\left(N_{\theta}=18\right.$ and $\left.N_{\theta}=9\right)$ are performed. Results are compared using SSIM to estimate the degradation encountered by the 


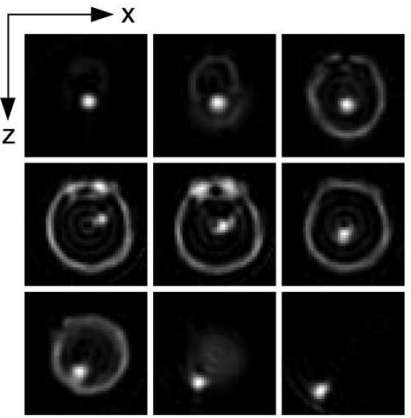

(a)

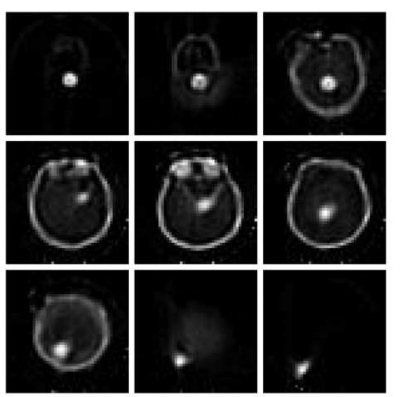

(b)

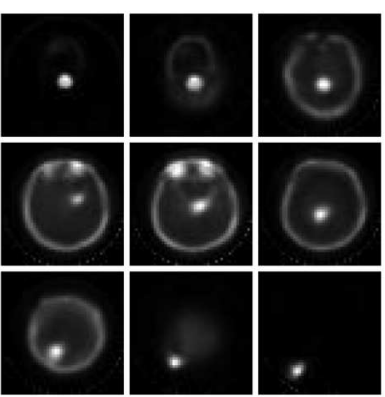

(c)

Fig. 6 Reconstructed cross-sectional images with optimized BFP (a), SART (b), and OSEM (c) techniques.

reconstructions. Results are given in Table 1. We can remark that the BFP quality decreases significantly with the projection number, whereas it remains quasi-constant with iterative techniques. These results correspond to the theoretical investigation. ${ }^{18}$ The Gaussian beam application in BFP does not reduce degradation and confirms the interest and efficiency of iterative techniques to reconstruct from only a few projections. Moreover, the Gaussian beam use has no bad impact on the projection number sensitivity whatever the method. Indeed, for a given method and fixed $N_{\theta}$, there is no significant difference between the SSIM degradation of the usual technique and the SSIM degradation of the optimized technique.

Now, we estimate the accuracy given by the optimized methods. The images in Fig. 7 represent a superposition of usual and optimized reconstruction results for each method. Red pixels correspond to the correction of extra pixels of the skull contour between usual and optimized techniques. They highlight that non-optimized methods suffer from a global spreading. Horizontal profiles of the sword [from the last images in Figs. 4(a)-4(c) and 6(a)-6(c)] are compared in Fig. 8 for each method. We can denote the lesser spreading of the metallic rod in the optimized reconstructions, especially with iterative techniques.

Finally, from 3-D vizualisations given in Fig. 9 (for optimized reconstructions only), we can perform several analyses such as the estimation of the skull boundary box size. From the usual techniques, we obtain a boundary volume of, $33 \times 39 \times 38 \mathrm{~mm}^{3}, 34 \times 36 \times 37 \mathrm{~mm}^{3}$, and $34 \times$ $36 \times 37 \mathrm{~mm}^{3}$ for BFP, SART, and OSEM, respectively. From the optimized techniques, we measure a boundary volume of $32 \times 36 \times 37 \mathrm{~mm}^{3}, \quad 31 \times 36 \times 35 \mathrm{~mm}^{3}$, and $33 \times 35 \times 37 \mathrm{~mm}^{3}$, for optimized BFP, SART, and OSEM, respectively. These results are closer to the real boundary box size of the skull $\left(30 \times 34 \times 37 \mathrm{~mm}^{3}\right)$ : with usual techniques, the size is overestimated along the $X$-axis and $Y$-axis between +2 to $+5 \mathrm{~mm}$, whereas it is no more than $\pm 2 \mathrm{~mm}$, with optimized methods (except $+3 \mathrm{~mm}$ along the $X$-axis with OSEM). However, we can denote a volume erosion $(-2 \mathrm{~mm})$ along the $Z$-axis with optimized SART. The total boundary box volume is overestimated by $+8 \mathrm{~mm}^{3}$ with usual techniques, whatever the method, whereas it is

Table 1 Comparison of SSIM degradation according to the usual and optimized (Opt.) techniques and the number of projections.

\begin{tabular}{llccccc}
$N_{\theta}$ & BFP & Opt. BFP & SART & Opt. SART & OSEM & Opt. OSEM \\
\hline 36 & 1 & 1 & 1 & 1 & 1 & 0.99 \\
18 & 0.98 & 0.99 & 0.98 & 0.97 & 0.99 \\
9 & 0.90 & 0.89 & 0.97 & 0.96 & 0.98 & 0.98 \\
\hline \hline
\end{tabular}

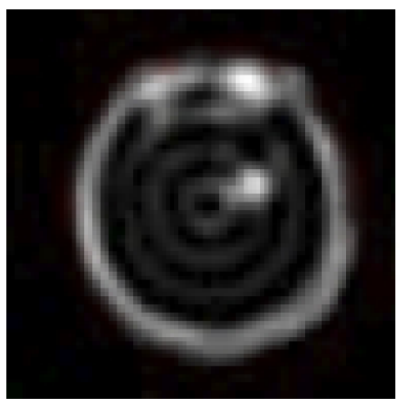

(a)

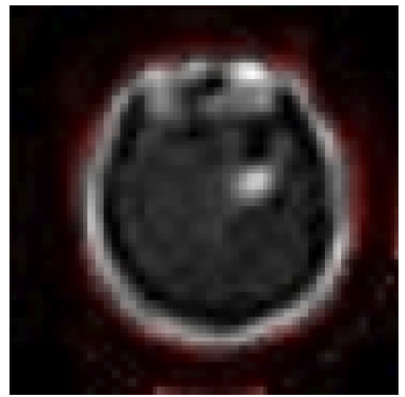

(b)

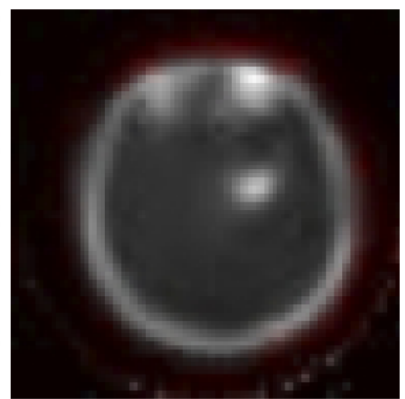

(c)

Fig. 7 Superposition of BFP and optimized BFP (a), SART and optimized SART (b), and OSEM and optimized OSEM (c) images at position $y=25$. The pixels colored in red correspond to the extra estimated skull contour in the non-optimized techniques. 

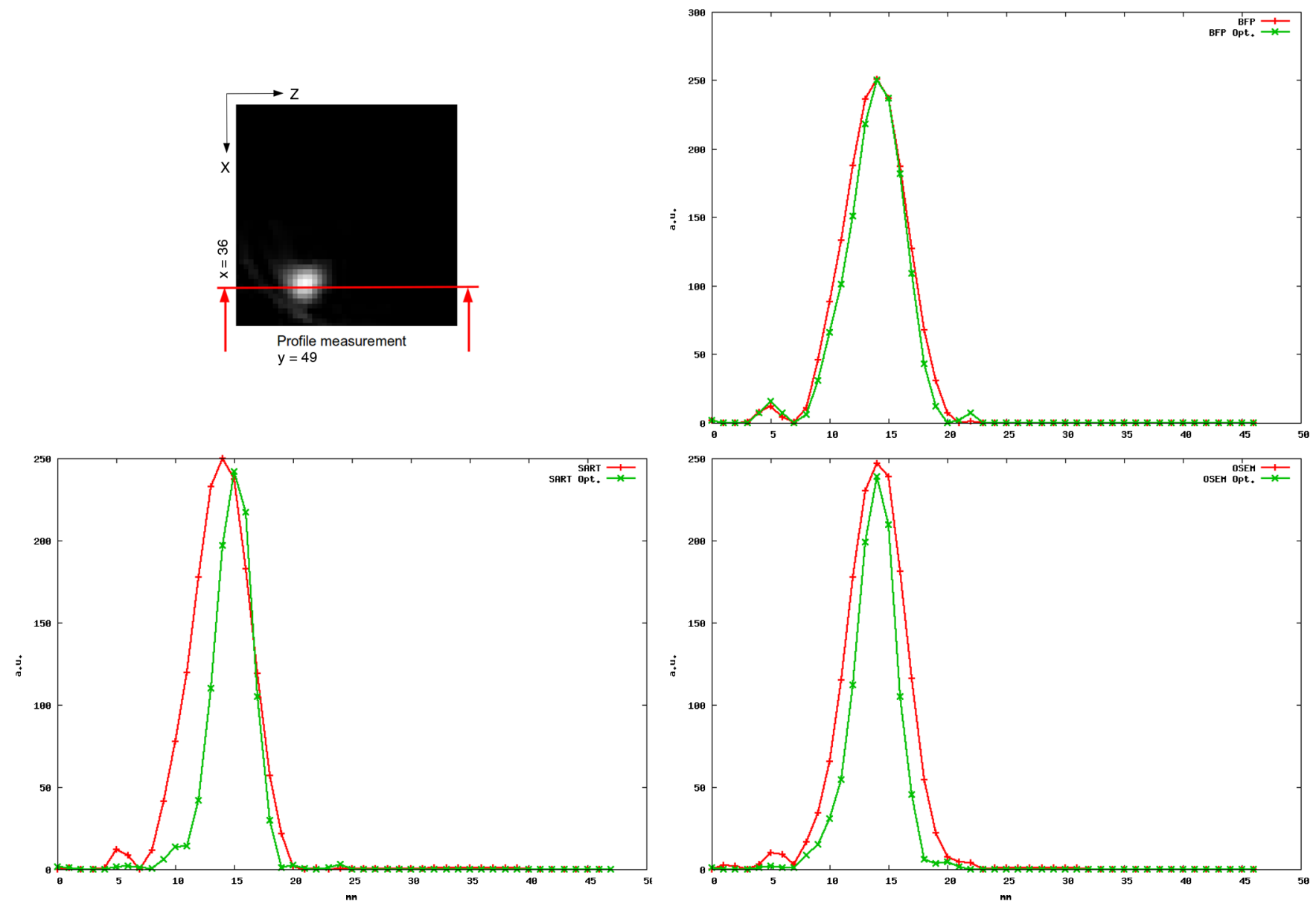

Fig. 8 Comparison of the horizontal profile of the metallic bar.

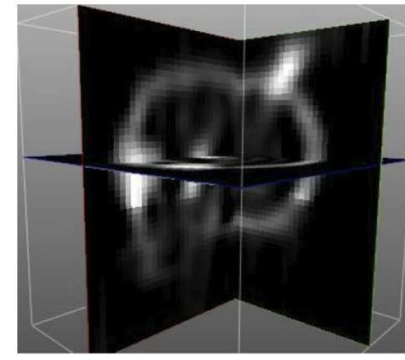

(a)

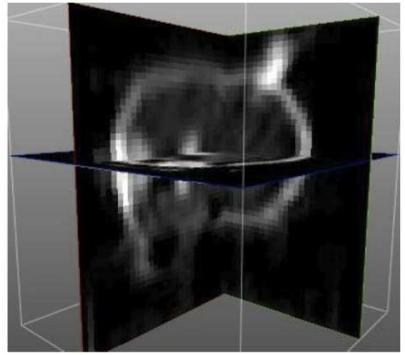

(b)

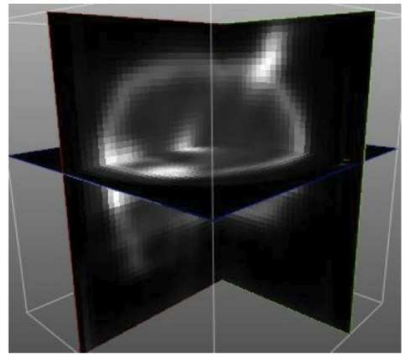

(c)

Fig. 9 3-D visualisation of the optimized BFP (a), SART (b), and OSEM (c) results.

overestimated only by $+4 \mathrm{~mm}^{3},-4 \mathrm{~mm}^{3}$, and $3 \mathrm{~mm}^{3}$ with optimized BFP, SART, and OSEM, respectively. According to these observations, the optimization proposed in this paper should be used preferably with the OSEM technique.

\section{Conclusion}

In this paper, we demonstrate an improved beam propagation model taking into account the physical properties of $\mathrm{THz}$ waves used in $\mathrm{THz}$ CT scan imaging. This more realistic model is clearly justified by the fact that previous models were simply relying on well-known and commonly applied $\mathrm{X}$-ray reconstruction methods, which assume a ray tracing approach, whereas the $\mathrm{THz}$ beam profile is very far from this hypothesis. The model is introduced in several inversion methods as a convolution filter to perform efficient tomographic reconstructions of acquired objects.

Results obtained with three different reconstruction methods (BFP, SART, and OSEM) have been compared on the basis of the optimized algorithm efficiency. We demonstrate an increase of the overall quality and accuracy of the reconstructions using image superpositions, profile analysis, and 3-D measurements. Moreover, we verify that the optimization has no bad effect on the efficiency of iterative techniques to reconstruct from a small number of projections. This approach has to be enforced by a modeling of the other 
physical properties of $\mathrm{THz}$ radiation (refraction and diffusion for instance) and has to be associated with a detector array sensor (THz camera) to provide a new improvement of 3-D THz CT imaging.

\section{Acknowledgments}

The authors are grateful for the financial support of this project in the frame of the DOTNAC program (Development and Optimization of THz NDT of Aeronautics Composite multilayered structures). Contract N 266320/FP7-AAT2010-RTD-1-http://www.dotnac-project.eu/.

\section{References}

1. D. M. Mittleman, R. H. Jacobsen, and M. C. Nuss, "T-ray imaging," IEEE J. Sel. Top. Quant. Electron. 2(3), 679-692 (1996).

2. A. El Fatimy et al., "Plasma wave field effect transistors as a resonant detector for imaging applications up to one terahertz for terahertz imaging," Opt. Commun. 282(15), 3055-3058 (2009).

3. E. Abraham et al., "Broadband terahertz imaging of documents written with lead pencils," Opt. Commun. 282(15), 3104-3107 (2009).

4. B. Ferguson et al., "T-ray computed tomography," Opt. Lett. 27(15), 1312-1314 (2002).

5. S. Wang et al., "T-ray imaging and tomography," J. Biol. Phys. 29(2-3), 247-256 (2003)

6. S. Wang and X. C. Zhang, "Pulsed terahertz tomography," J. Phys. D: Appl. Phys. 37(4), R1-R36 (2004).

7. M. M. Awad and R. A. Cheville, "Transmission terahertz waveguidebased imaging below the diffraction limit," Appl. Phys. Lett. 86(22), 221107 (2005).

8. X. Yin et al., "Wavelet based local tomographic image using terahertz techniques," Digit. Signal Process. 19(4), 750-763 (2009).
9. A. Brahm et al., "Volumetric spectral analysis of materials using terahertz-tomography techniques," Appl. Phys. B 100(1), 151-158 (2010).

10. E. Abraham et al., "Refraction losses in terahertz computed tomography," Opt. Commun. 283(10), 2050-2055 (2010).

11. P. Toft, "The radon transform : theory and implementation," $\mathrm{Ph}$. D. Thesis, Department of Mathematical Modelling, Section for Digital Signal Processing, Technical University of Denmark(1996).

12. J. Radon, "Über die Bestimmung von Funktionen durch ihre Integralwerte längs gewisser Mannigfaltigkeiten,”Ber. Ver. Sachs. Akad. Wiss. Leipzig, Math-Phys. Kl 69, 262-277 (1917).

13. R. Gordon, R. Bender, and G. T. Herman, "Algebraic reconstruction techniques (ART) for three-dimensional electron microscopy and x-ray photography," J. Theor. Biol. 29(3), 471-481 (1970).

14. A. H. Andersen and A. C. Kak, "Simultaneous algebraic reconstruction technique (SART): a superior implementation of the ART algorithm," Ultrason. Imag. 6(1), 81-94 (1984).

15. L. A. Shepp and Y. Vardi, "Maximum likelihood reconstruction for emission tomography," IEEE Trans. Med. Imag. 1(2), 113-122 (1982).

16. H. M. Hudson and R. S. Larkin, "Accelerated image reconstruction using ordered subsets of projection data," IEEE Trans. Med. Imag. 13(4), 601-609 (1994).

17. G. T. Herman, Image Reconstruction From Projections : The Fundamentals of Computerized Tomography, Academic Press Inc., New York (1980).

18. B. Recur et al., "Investigation on reconstruction methods applied to 3D terahertz computed tomography," Opt. Express 19(6), 5105-5117 (2011).

19. B. Recur et al., "Propagation beam consideration for 3D Thz computed tomography," accepted for publication in Opt. Express 20(6), 5817-5892 (2011).

20. Z. Wang et al., "Image quality assessment : from error visibility to structural similarity," IEEE Trans. Image Process. 13(4), 600-612 (2004)

Biographies and photographs of the authors are not available. 